\title{
A new planar defect in SiGe nanopillars
}

Hongbin Yang ${ }^{1}$, Shang Ren ${ }^{2}$, Emily Turner ${ }^{3}$, Sobhit Singh $^{2}$, Kevin Jones ${ }^{3}$, Philip Batson ${ }^{2}$, David Vanderbilt $^{2}$ and Eric Garfunkel ${ }^{4}$

${ }^{1}$ Department of Chemistry and Chemical Biology, Rutgers University, Piscataway, New Jersey, United

States, ${ }^{2}$ Department of Physics and Astronomy, Rutgers University, United States, ${ }^{3}$ Department of

Materials Science and Engineering, University of Florida, United States, ${ }^{4}$ Department of Chemistry and Chemical Biology, Rutgers University, United States

Planar defects exist in many diamond-type semiconductors. Experimentally, they have been found in the $\{001\}$ planes in both diamond and Ge, but with distinct atomic arrangements ${ }^{1,2}$. In Si and SiGe, planar defects are known to exist mainly in the $\{113\}$ and occasionally $\{111\}$ planes $^{3}$, but not in the $\{001\}$ planes. The structural and electronic properties, as well as formation mechanism of these defects, are of great interest because of the importance of these materials in electronic devices.

Here we present a new planar defect observed in the (001) plane in a SiGe nanostructure. Atomicresolution imaging of this defect has been carried out by an aberration-corrected scanning transmission electron microscope (STEM) operating at $60 \mathrm{kV}$, below the damage threshold ${ }^{3}$. The defect viewed along the [110] projection is shown in Fig. 1(b), which suggests that the structural model originally proposed by Humble for diamond ${ }^{4}$ applies to this defect. Within this model, shown in Fig. 1(a), all atoms are 4fold coordinated but experience large bond angle distortion. The tilted dumbbells in Fig. 1(b) closely resemble the $2 \times 1$ reconstructed (001) surface of a diamond cubic crystal, where the atoms on the surface form symmetric dimers. Dimerization also occurs for the upper layer of the purple atoms, but in the direction parallel to the electron beam. For an extended two-dimensional defect, there is more than one way this reconstruction can occur and give rise to the structure we see in Fig. 1(b) ${ }^{5}$. To fully determine structure, we have also imaged the same planar defect along the [210] direction, shown in Fig. 1(c). Comparing this image with the structural models proposed by Goss et $\mathrm{al}^{5}$, we have determined the exact atomic arrangement of this defect in three dimensions. This planar defect in SiGe has a structure that is different from both the $\{001\}$ platelets in diamond ${ }^{1}$ and the theoretically predicted most stable $\{001\}$ defects in $\mathrm{Si}^{6}$.

In prior work on planar defects in $\mathrm{Si}$ and $\mathrm{Ge}$, the creation process usually involved ion/high energy electron irradiation ${ }^{2,3}$. In our work, the defect and nanostructure were formed by oxidation of a patterned $\mathrm{Si} / \mathrm{SiGe}$ superlattice, similar to the process described in Brewer et $\mathrm{al}^{7}$. After oxidation of a SiGe nanopillar, a planar defect form across the partially oxidized pillar, see Fig. 2(a) and (b). The Si content of the SiGe pillar has been characterized by electron energy loss spectroscopy (EELS) across the planar defect with high spatial resolution, shown in Fig. 2(c) and (d). Very close to the defect, we see a significant decrease in Si content. This suggests that the defect occurs with predominantly Ge-Ge bonding. 
(a)

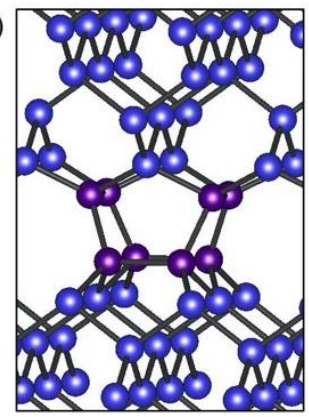

(b)

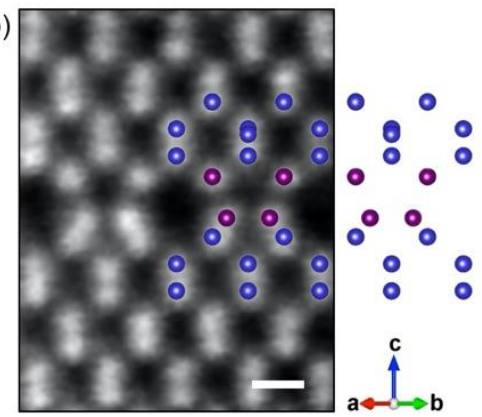

(c)

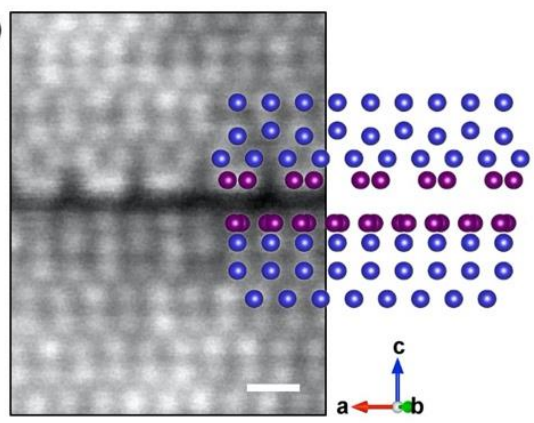

Figure 1. (a) A modified Humble model of a $\{001\}$ defect inside a diamond cubic lattice. Atoms shown in purple indicate the atoms that are under significant bond angle distortion. All atoms in the model are either Si or Ge. (b) Atomic-resolution HAADF-STEM images of the defect viewed from [110] projection and (c) from [210] projection, with the structural models shown superimposed. Scalebar = $3 \AA$.

(a)

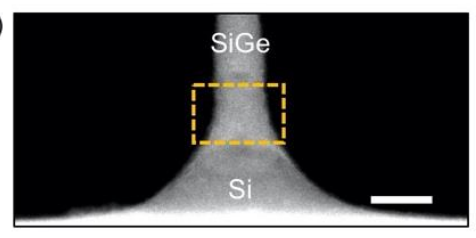

(b)

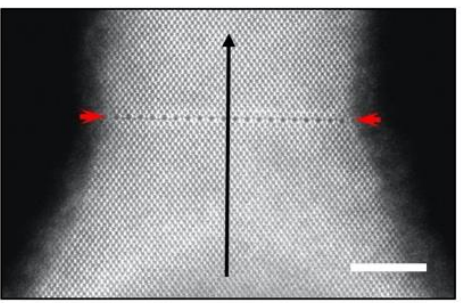

(c)

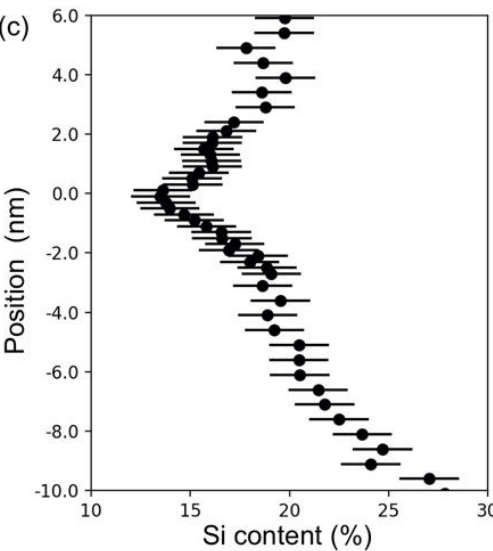

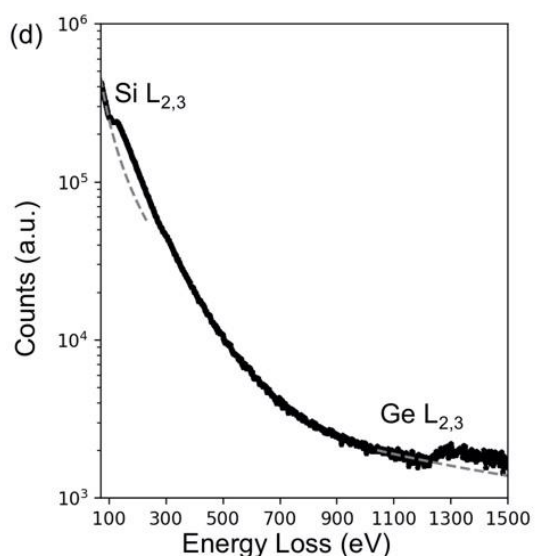

Figure 2. (a) Cross-sectional HAADF-STEM image of a Si/SiGe pillar on a Si substrate, scalebar = 20nm. (b) Zoomed-in view of the boxed area in (a), the planar defect is indicated by two red arrows, scalebar $=5 \mathrm{~nm}$. (c) Line profile of Si content along the black arrow in (b), quantified via EELS of Si and Ge $\mathrm{L}_{2,3}$ edges as shown in $(\mathrm{d})$.

References

1. E. J. Olivier, J. H. Neethling, R. E. Kroon, S. R. Naidoo, C. S. Allen, H. Sawada, P. A. van Aken and A. I. Kirkland, Nat. Mater. 17 (3), 243-248 (2018).

2. S. Muto and S. Takeda, Phil. Mag. Lett. 72 (2), 99-104 (1995).

3. L. Fedina, O. I. Lebedev, G. Van Tendeloo, J. Van Landuyt, O. A. Mironov and E. H. C. Parker, Phys. Rev. B 61 (15), 10336-10345 (2000).

4. P. Humble, Proc. R. Soc. London, Ser. A. 381 (1780), 65-81 (1982).

5. J. P. Goss, T. A. G. Eberlein, R. Jones, N. Pinho, A. T. Blumenau, T. Frauenheim, P. R. Briddon and S. berg, J. Phys.: Condens. Matter. 14 (48), 12843-12853 (2002).

6. J. P. Goss, B. J. Coomer, R. Jones, C. J. Fall, P. R. Briddon and S. Öberg, Phys. Rev. B 67 (16) (2003). 